\title{
Japan digs deep for secrets of the Sun
}

Kamioka, Japan. A vast chasm 40 metres in diameter and 40 metres deep is being cut out deep under a mountain in the centre of Japan to accommodate a detector which, its designers hope, will answer one of the key questions facing modern cosmologists: why does the number of neutrinos observed from the Sun seem to be about half that predicted by theory.

The world's largest neutrino detector, the Super-Kamiokande observatory, is currently under construction $1 \mathrm{~km}$ down a zinc-and-lead mine known as the Kamioka mine. The detector is being built with total funding of grant of $¥ 10$ billion ( $\$ 95$ million) from Japan's Ministry of Education, Science and Culture, and should be ready for testing in two years time.

When neutrinos enter a tank of water, they occasionally collide with water

\section{UK plans library of DNA profiles}

London. Britain's Home Secretary, Michael Howard, announced last week that the government intends to establish a library containing the DNA profiles of virtually anyone convicted of committing a criminal offence.

The move, which will put DNA profiling on the same level as conventional fingerprinting, goes considerably further than the recent recommendation of the Royal Commission on Criminal Justice. This had suggested that a national DNA library should be restricted only to those convicted of relatively serious criminal offences, such as burglary and assault.

The Royal Commission's suggestion would have lead to a library of about 180,000 profiles. The government's decision to go for a larger grouping will result in one containing 500,000 , almost one in ten of Britain's population.

The government's decision was one of 27 new measures announced by Howard last week at the Conservative Party's annual conference in Blackpool designed to demonstrate the party's commitment to law and order. "DNA will be for today's policeman what fingerprints were for Dixon of Dock Green", said Howard, referring to the hero of a popular television series in the 1950s and 1960 s.

Critics of DNA profiling, however, continue to warn of the need for caution in introducing its widespread use while questions about the accuracy and statistical validity of the tests remain in dispute. molecules, creating very faint Cerenkov radiation which can be picked up by photomultiplier tubes in the sides of the tank. Using such techniques, the smaller Kamiokande observatory in the

same mine made headlines in 1987 when it detected neutrinos from a supernova in the Large Magellanic Cloud.

The new detector, Super-Kamiokande, will be more than 10 times the size of Kamiokande. Containing 50,000 tonnes of water, it should be able to detect about 30 solar neutrinos a day instead of only one every three days caught by the existing detector. Yoji Totsuka of Tokyo University's Institute for Cosmic Ray Research, who is leading the project, says that this larger detection rate will enable the observatory to help resolve the 20-year controversy over solar neutrinos.

The detector is located deep in the mine to filter out cosmic radiation that would disturb the observation of neutrinos. It runs largely automatically, recording events on magnetic tape.

But operating it still requires dedication from scientists. Kamiokande is reached by a 15-minute ride in an old tram down a slanting mine-shaft in pitch darkness. One scientist from Totsuka's group, assigned for an eight-day shift, monitors the machine during the day with only one tram in the morning and one out at night.

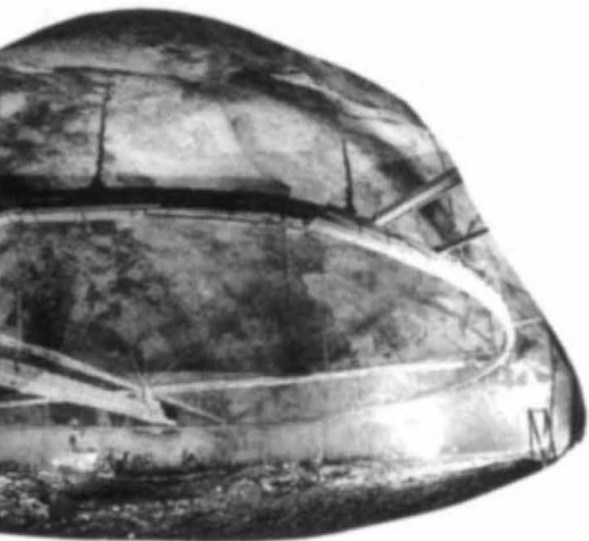

Under construction - the SuperKamiokande observatory.

Just over a quarter of the construction money will be spent on the manufacture of 11,200 highly sensitive 20 -inch photomultiplier tubes that will line the walls of the giant detector. Totsuka says he was able to negotiate a 60 per cent discount on the normal price of the tubes from Hamamatsu Photonics, a small Japanese company that is the world's only manufacturer of such tubes.

A similar sum is being paid to Kamioka Mining and Smelting Company, owner of the mine, for excavation and construction work. The contract is partly a reward to the company for supporting the earlier Kamiokande project which was built on a shoestring budget of only $¥ 500$ million.

Excavation of the cavern to accommodate the detector will be completed by next August. The detector itself, as well as a water purification system and other facilities such as a network of workstations for collecting and analysing data, should be in place by October 1995, in time for routine observations to begin in the spring of 1996 .

David Swinbanks

\section{Mine finds uses for "inner space"}

Kamiokande and Super-Kamiokande are not the only scientific experiments being carried out in the Kamioka mine, one of the largest and most modern zinc-lead mines in the world. The rapid rise in the value of the yen has reduced annual sales from about $¥ 30$ to $¥ 17$ billion. As a result, the company that operates the mine has been looking at a number of ways of exploiting it for scientific purposes.

In one experiment, an explosives company is renting a tank of water for carrying out underwater tests on new explosives. And a scientist from Tokyo University is helping to develop a large chamber for carrying out controlled tests on athletes under high-altitude conditions.

The athletes are sealed in the chamber, and sufficient air is sucked out to simulate high-altitude conditions up to the equivalent of 4,000 metres. The athletes use exercisers while their physiological condition is monitored.

The high-altitude chamber, built at a cost of $¥ 40$ million, is still at the test stage. But officials at the mine are hoping that companies interested in supporting Olympic athletes will invest money in a larger facility.

At present, such scientific activities including the construction of SuperKamiokande - account for about five per cent of the company's income. But with the mine only expected to be viable for about another 15 years, officials hope that what they describe as "inner space technology" will provide a growing source of revenue. 